\title{
ABDELILAH GMIRA \\ Existence de la trace initiale pour des solutions d'une équation parabolique dégénérée
}

\author{
Annales de la faculté des sciences de Toulouse $5^{e}$ série, tome $8, \mathrm{n}^{\circ} 3$ \\ (1986-1987), p. 315-329 \\ <http://www.numdam.org/item?id=AFST_1986-1987_5_8_3_315_0>
}

○) Université Paul Sabatier, 1986-1987, tous droits réservés.

L'accès aux archives de la revue «Annales de la faculté des sciences de Toulouse » (http://picard.ups-tlse.fr/ annales/) implique l'accord avec les conditions générales d'utilisation (http://www.numdam.org/conditions). Toute utilisation commerciale ou impression systématique est constitutive d'une infraction pénale. Toute copie ou impression de ce fichier doit contenir la présente mention de copyright.

\section{Numdam}

Article numérisé dans le cadre du programme

Numérisation de documents anciens mathématiques

http://www.numdam.org/ 


\title{
Existence de la trace initiale pour des solutions d'une équation parabolique dégénérée
}

\author{
AbDelilah Gmira(1) (2)
}

Résume. - On démontre l'existence et l'unicité de la trace initiale pour chaque solution positive de l'équation $u_{t}=\operatorname{div}\left(|\nabla u|^{p-2} \nabla u\right)$ dans $\left.\mathbf{R}^{N} \times\right] 0, T[$. De plus on prouve que la trace doit vérifier une certaine condition de croissance; d'où on déduit que la moyenne sur la boule $B_{R}(0)$ de $u(x, 0)$ ne doit pas croître plus vite que $|x|^{p / p-2}$ quand $|x| \rightarrow+\infty$.

ABstract. - We show the existence of an unique trace of each nonnegative solution of the equation $u_{t}=\operatorname{div}\left(|\nabla u|^{p-2} \nabla u\right)$ in $\left.\mathbf{R}^{N} \times\right], T[$. And we prove that the trace must satisfy some growth condition; then we deduce that the average on the ball $B_{R}(0)$ of $u(x, 0)$ cannot grow faster than $|x|^{p / p-2}$ as $|x| \rightarrow+\infty$.

\section{Introduction}

L'objet de cet article est d'étudier l'existence et l'unicité de la trace initiale de solutions non négatives de l'équation parabolique non linéaire :

$$
u_{t}=\operatorname{div}\left(|\nabla u|^{p-2} \nabla u\right) ; p \geq 2
$$

$\left.\operatorname{sur} \Omega_{T}=\mathbf{R}^{N} \times\right] 0, T[$.

Si $p=2$, on obtient l'équation de la chaleur :

$$
u_{t}=\nabla u .
$$

(1) Faculté des Sciences et des Techniques, Laboratoire de Mathématiques 16, route de Gray - 25030 Besançon Cédex

(2) Faculté des Sciences, B.P. 300 - Tétouan (Maroc) 


\section{A. Gmira}

Aronson dans [1], en utilisant la représentation de Widder, démontre l'existence et l'unicité d'une mesure de Borel $\mu$ non négative telle que

$$
\lim _{t \searrow 0} \int_{\mathbf{R}^{N}} u(x, t) \varphi(x) d x=\int_{\mathbf{R}^{N}} \varphi(x) \mu(d x)
$$

pour toute fonction $\varphi \in C_{o}\left(\mathbf{R}^{N}\right)$.

C'est-à-dire que toute solution $u$ non négative de $(0.2)$ admet une unique mesure de Borel $\mu$ comme trace initiale, au sens de (0.3). De plus il prouve que $\mu$ est $\sigma$-finie et elle vérifie

$$
\int_{\mathbf{R}^{N}} e^{-|\xi|^{2} / 4 t} \mu(d x)<+\infty .
$$

Si $p>2$, l'équation (0.1) apparaît en théorie des fluides non newtoniens (voir LeE et AMEs [4]). De plus de telles équations sont un prototype pour certaines classes d'équations dégénérées.

En utilisant des solutions de similarité explicites de l'équation (0.1), et une inégalité d'Harnack faible, on démontre l'existence et l'unicité d'une mesure de Borel $\mu$ qui est trace initiale pour chaque solution de (0.1). De plus on prouve que $\mu$ vérifie l'estimation

$$
\int_{B_{R}(0)} \mu(d x) \leq C\left\{R^{k / p-2} T^{-1 / p-2}+T^{N / p} u^{k / p}(0, T)\right\}
$$

où $k=p+N(p-2), C$ étant une constante positive dépendant de $N$ et $p$, pour tout $R \in \mathbf{R}^{+}$. C'est-à-dire que la moyenne $\int_{B_{R}(0)} u(x, 0) d x$ de $u(x, 0)$ sur $B_{R}(0)$ ne peut pas croître plus vite que $|x|^{\frac{p}{p-2}}$ quand $|x| \rightarrow+\infty$.

L'article va être divisé en 2 parties :

Dans la lère partie, on donne un principe de comparaison locale des solutions de l'équation (0.1).

Quant à la 2ème partie, elle est consacrée à l'existence et à l'unicité de la trace initiale.

\section{Préliminaires}

Dans cette partie, on va donner quelques propriétés des solutions faibles de l'équation

$$
\begin{aligned}
u_{t}=\operatorname{div} & {\left[|\nabla u|^{p-2} \nabla u\right] } \\
- & 316-
\end{aligned}
$$


Existence de la trace initiale

où $p>2$.

DÉFINITION 1.1. - Une solution du problème (1.1) dans $\Omega_{T}=\mathbf{R}^{N} \times$ $(0, T)$ est une fonction $u=u(x, t)$ définie et continue sur $\Omega_{T}$ telle que :

(i) $\nabla u \in L_{l o c}^{p}\left(\Omega_{T}\right)$,

(ii) Pour toute fonction $\varphi$ à support compact dans $\Omega_{T}$ dont les dérivées $\varphi_{t} \in L^{1}\left(\Omega_{T}\right)$ et $\nabla_{\varphi} \in L^{p}\left(\Omega_{T}\right)$, on $a$ :

$$
\int_{0}^{T} \int_{\mathbf{R}^{N}}\left[u \varphi_{t}-|\nabla u|^{p-2} \nabla u \nabla \varphi\right] d x d t=0
$$

Pour donner un principe de comparaison locale des solutions de l'équation (1.1), on a besoin d'introduire un problème tronqué de (1.1). Ainsi, pour tout $x_{o} \in \mathbf{R}^{N}, R \in \mathbf{R}^{+}$et $t_{1}, t_{2}: 0<t_{1}<t_{2} \leq T$. On pose $\left.S=B_{R}\left(x_{o}\right) \times\right] t_{1}, t_{2}[$ et $\partial_{p} S=\bar{S} \backslash S$ étant la frontière parabolique où $B_{R}\left(x_{o}\right)$ est la boule de centre $x_{o}$ et de rayon $R$. On considère le problème

$$
\begin{cases}v_{t}=\operatorname{div}\left[|\nabla v|^{p-2} \nabla v\right] & \text { dans } S \\ V=g & \text { sur } \partial_{p} S\end{cases}
$$

DÉFINITION 1.2.- Une solution du problème (1.3) est une fonction $V=V(x, t)$ définie et continue sur $\bar{S}$ telle que :

(i) $\nabla V \in L^{p}(S)$

(ii) $V=g$ sur $\partial_{p} S$

(iii) Pour toute fonction $\varphi$ à support compact dans $S$ dont les dérivées $\varphi_{t} \in L^{1}(S)$ et $\nabla \varphi \in L^{p}(S)$, on a :

$$
\int_{t_{1}}^{t_{2}} \int_{B_{R}\left(x_{o}\right)}\left(V \varphi_{t}-|\nabla V|^{p-2} \nabla V \nabla \varphi\right) d x d t=0
$$

Les solutions de l'équation (1.3) vérifient le principe de comparaison locale suivant :

Proposition 1.1. - Soit $V_{i}(i=1,2)$ la solution faible du problème (1.9), dont la donnée sur la frontière parabolique $\partial_{p} S$ est $g_{i}$.

Si $g_{1} \leq g_{2}$ sur $\partial_{p} S$, alors $V_{1} \leq V_{2}$ sur $\bar{S}$. 


\section{A. Gmira}

Preuve. - On considère la suite de fonctions $\left(f_{n}\right)_{n \in \mathbb{N}}$ définie sur $[0, R]$ à valeurs dans $\mathbf{R}^{+}$par :

$$
\left\{\begin{array}{l}
0 \leq f_{n} \leq 1, \\
f_{n}(r)=1 \\
f_{n} \in \mathcal{D}([0, R]) .
\end{array} \text { si } r \in\left[0, R-\frac{1}{n}\right]\right.
$$

On se donne un point quelconque $t \in\left(t_{1}, t_{2}\right)$, et puis on définit la suite de fonctions $\left(Z_{k}\right)_{k \in \mathbb{N}}$ par :

$$
Z_{k}(s)= \begin{cases}1 & \text { sur }\left[t_{1}, t-\frac{1}{k}\right] \\ k(t-s) & \text { sur } \left.] t-\frac{1}{k}, t\right] \\ 0 & \text { sur } \left.] t, t_{2}\right] .\end{cases}
$$

On pose $V=V_{1}-V_{2}$.

Soit la fonction $\varphi$ définie $\operatorname{sur}\left(t_{1}, t_{2}\right) \times B_{R}\left(x_{o}\right)$ par :

$$
\varphi(x, s)=Z_{k}(s) f_{n}\left(\left|x-x_{o}\right|\right) \int_{s}^{s+h} \frac{V^{+}(x, \mathcal{I})}{h} d \mathcal{I} .
$$

D'où on obtient

$$
\begin{aligned}
\frac{\partial \varphi}{\partial t}(x, s) & =Z_{k}^{\prime}(s) f_{n}\left(\left|x-x_{o}\right|\right) \int_{s}^{s+h} h^{-1} V^{+}(x, \mathcal{I}) d \mathcal{I} \\
& +Z_{k}(s) f_{n}\left(\left|x-x_{o}\right|\right) \nabla_{h}\left(V^{+}(x, s)\right)
\end{aligned}
$$

où

$$
\begin{aligned}
\nabla_{h}\left(V^{+}(x, s)\right)=h^{-1}\left[V^{+}(x, s+h)-V^{+}(x, s)\right] \\
\nabla \varphi(x, s)=Z_{k}(s) \nabla f_{n}\left(\left|x-x_{o}\right|\right) \int_{s}^{s+h} h^{-1} V^{+}(x, \mathcal{I}) d \mathcal{I} \\
+Z_{k}(s) f_{n}\left(\left|x-x_{o}\right|\right) \int_{s}^{s+h} h^{-1} \nabla V^{+}(x, \mathcal{I}) d \mathcal{I} .
\end{aligned}
$$

Donc $\varphi$ est à support compact dans $S$; de plus $\varphi_{t} \in L^{1}(S)$ et $\nabla \varphi \in L^{p}(S)$. Par conséquent on a :

$$
\int_{t_{1}}^{t_{2}} \int_{B_{R}\left(x_{o}\right)}\left[V_{i} \varphi_{t}-\left|\nabla V_{i}\right|^{p-2} \nabla V_{i} \nabla \varphi\right] d x d t=0
$$




\section{Existence de la trace initiale}

pour $i=1,2$.

En faisant la différence des deux équations, on obtient :

$$
\begin{aligned}
& \int_{t_{1}}^{t_{2}} \int_{B_{R}\left(x_{0}\right)} V \varphi_{t} d x d t= \\
& \int_{t_{1}}^{t_{2}} \int_{B_{R}\left(x_{0}\right)}\left[\left|\nabla V_{1}\right|^{p-2} \nabla V_{1}-\left|\nabla V_{2}\right|^{p-2} \nabla V_{2}\right] \nabla \varphi d x d t .
\end{aligned}
$$

Vu la relation $(1.8)$, on a

$$
\lim _{\substack{n \rightarrow+\infty \\ h \rightarrow 0}} \frac{\partial \varphi}{\partial t}(x, s)=Z_{k}^{\prime}(s) V^{+}(x, s)+Z_{k}(s) \frac{\partial}{\partial t}\left(V^{+}(x, s)\right) .
$$

En utilisant l'expression de $Z_{k}$, on obtient

$$
\begin{aligned}
& \lim _{k \rightarrow+\infty} \int_{t_{1}}^{t_{2}} \int_{B_{R}\left(x_{0}\right)} V(x, s) Z_{k}(s) \frac{\partial}{\partial t}\left(V^{+}(x, s)\right) d x d t= \\
& \quad=\int_{t_{1}}^{t} \int_{B_{R}\left(x_{0}\right)} V(x, s) \frac{\partial}{\partial t}\left(V^{+}(x, s)\right) d x d t=\frac{1}{2} \int_{B_{R}\left(x_{o}\right)} V^{+2}(x, t) d x
\end{aligned}
$$

$\left(\operatorname{car} V^{+}=0 \operatorname{sur} \partial_{p} S\right)$.

De même, on a :

$$
\begin{aligned}
& \lim _{k \rightarrow+\infty} \int_{t_{1}}^{t_{2}} \int_{B_{R}\left(x_{o}\right)} V(x, s) Z_{k}^{\prime}(s) V^{+}(x, s) d s= \\
& =-\int_{B_{R}\left(x_{o}\right)} V(x, t) \cdot V^{+}(x, t) d x=-\int_{B_{R}\left(x_{o}\right)} V^{+2}(x, t) d x .
\end{aligned}
$$

En utilisant l'expression de $\nabla \varphi$, on a

$$
\lim _{\substack{k \rightarrow+\infty \\ h \rightarrow 0}} \nabla \varphi(x, s)=\nabla f_{n}\left(\left|x-x_{o}\right|\right) V^{+}(x, s)+f_{n}\left(\left|x-x_{o}\right|\right) \nabla V^{+}(x, s) .
$$

Ainsi

$$
\begin{aligned}
& \lim _{\substack{n, k \rightarrow+\infty \\
h \rightarrow 0}} \int_{t_{1}}^{t_{2}} \int_{B_{R}\left(x_{0}\right)}\left[\left|\nabla V_{1}\right|^{p-2} \nabla V_{1}-\left|\nabla V_{2}\right|^{p-2} \nabla V_{2}\right] \nabla \varphi d x d t= \\
& =\int_{t_{1}}^{t_{2}} \int_{B_{R}\left(x_{0}\right)}\left[\left|\nabla V_{1}\right|^{p-2} \nabla V_{1}-\left|\nabla V_{2}\right|^{p-2} \nabla V_{2}\right] \nabla V^{+} d x d s \geq 0 .
\end{aligned}
$$




\section{A. Gmira}

Par conséquent, en utilisant les relations (1.14), (1.15) et (1.17) et puis en passant à la limite sur $n, k$ et $h$ dans la relation (1.12), on obtient

$$
\int_{B_{R}\left(x_{o}\right)} V^{+2}(x, t) d x \leq 0
$$

d'où on conclut que $V^{+}(x, t)=0$, pour tout $x \in B_{R}\left(x_{o}\right)$ (car $V$ est continue d'après [3]), c'est-à-dire

$$
V_{1}(\cdot, t) \leq V_{2}(\cdot, t), \quad \operatorname{sur} B_{R}\left(x_{o}\right)
$$

Mais comme ceci est vrai pour tout $t \in] t_{1}, t_{2}[$, alors on obtient

$$
\left.V_{1} \leq V_{2} \text { sur } B_{R}\left(x_{0}\right) \times\right] t_{1}, t_{2}[
$$

Du fait que $g_{1} \leq g_{2}$ sur $\partial_{p} S$, alors on déduit que $V_{1} \leq V_{2}$ sur $\bar{S}$.

Proposition 1.2. - Soit $u$ une solution faible dans $\left.\Omega_{T}=\mathbf{R}^{N} \times\right] 0, T[$ de l'équation (1.1). Pour tout cylindre $\left.S=B_{R}\left(x_{o}\right) \times\right] t_{1}, t_{2}\left[\right.$ contenu dans $\Omega_{T}$, $u$ est une solution faible du problème (1.3) dont la donnée sur la frontière parabolique $\partial_{p} S$ est la restriction de $u$ à $\partial_{p} S$.

La démonstration de la proposition est évidente, en utilisant le fait que $u$ est continue sur $\left.B_{R}\left(x_{o}\right) \times\right] t_{1}, t_{2}$ [ (d'après [3]).

Dans ce qui suit, on va donner une estimation du support de la solution de l'équation (1.3) quand la donnée sur la frontière $\partial_{p} S$ est à support compact; plus précisément on a la

Proposition 1.3. - Soit $w$ une solution faible du problème

$$
\begin{cases}w_{t}=\operatorname{div}\left[|\nabla w|^{p-2} \nabla w\right] & \text { dans } \left.\mathbf{R}^{N} \times\right] t_{1}, T[, \\ w\left(\cdot, t_{1}\right)=g & \text { dans } \mathbf{R}^{N} .\end{cases}
$$

où $0 \leq t_{1}<T$ et $g$ étant une fonction continue. Si $g$ est à support compact, alors il existe une constante $R>0$ telle que

$$
\text { Supp } w(\cdot, t) \subset B_{R} \text {, pour tout } t \in\left[t_{1}, T\right) \text {. }
$$

Démonstration. - Soit $r_{o}>0$ telle que Supp $g \subset B_{r_{o}}$. On pose

$$
B(x, t)=\frac{a}{R^{N}(t)}\left\{\left[1-\left(\frac{|x|}{R(t)}\right)^{\frac{p}{p-1}}\right]+\right\}^{\frac{p-1}{p-2}}
$$


où

$$
R(t)=\alpha t^{1 / k} ; k=p+N(p-2)
$$

$\alpha$ et $a$ étant fixés.

Pour tout $\lambda \in \mathbf{R}^{+}$, on considère la suite $\left(u_{\lambda}\right)_{\lambda \in \mathbf{N}^{*}}$ définie par

$$
u_{\lambda}(x, t)=\lambda^{-\frac{p}{p-2}} B(\lambda x, t) .
$$

Il est évident que $u_{\lambda}$ est une solution faible de l'équation (1.1). De plus on a les estimations suivantes

$$
\begin{gathered}
\text { Supp } u_{\lambda}(\cdot, t) \subset B_{R}(0), \\
R=\left(\frac{\alpha}{\lambda}\right) T^{1 / k} .
\end{gathered}
$$

On considère $w_{R}$ la solution du problème (qui existe et qui est unique car $g$ est continue) du problème :

$$
\begin{cases}\left(W_{R}\right)_{t}=\operatorname{div}\left[\left|\nabla W_{R}\right|^{p-2} \nabla W_{R}\right] & \text { dans } \left.S_{R}=B_{R} \times\right] t_{1}, T[ \\ W_{R}=g & \text { sur } \partial_{p} S_{R} .\end{cases}
$$

Comme pour tout $t>0, u_{\lambda}(\cdot, t)$ tend vers l'infini quand $t$ tend vers 0 ; et de plus Supp $u_{\lambda}(\cdot, t) \rightarrow \mathbf{R}^{N}$ quand, $t \rightarrow+\infty$. On peut supposer que $\lambda$ assez petit, tel que

$$
g \leq u_{\lambda} \operatorname{sur} \partial_{p} S_{R}
$$

Ainsi d'après la proposition 1.1 , on a

$$
W_{R} \leq u_{\lambda} \operatorname{sur} \bar{S}_{R}
$$

Du fait de l'unicité de la solution du problème (1.28), on déduit que $W_{R}$ est à support compact et même Supp $W_{R} \subset B_{R}(0)$.

Mais comme $g$ est continue, alors le problème (1.21) admet une unique solution; et en utilisant la proposition 1.2, on déduit que $w$ est aussi à support compact; de plus

$$
\text { Supp } w(\cdot, t) \subset B_{R}(0), \text { pour tout } t \in\left[t_{1}, T[.\right.
$$

Le dernier résultat de ce paragraphe est un principe de comparaison globale; plus précisément : 


\section{A. Gmira}

Proposition 1.4. - Soit u une solution faible non négative du problème (1.1) dans $\mathbf{R}^{N} \times(0, T)$. Pour chaque fonction $z \in C_{o}^{\infty}\left(\mathbf{R}^{N}\right)$ à valeurs dans $[0,1]$, et pour tout $\mathcal{I} \in(0, T)$, soit $w$ la solution du problème

$$
\begin{cases}w_{t}=\operatorname{div}\left[|\nabla w|^{p-2} \nabla w\right] & \text { dans } \left.\left.R^{N} \times\right] \mathcal{I}, T\right) \\ w(x, \mathcal{I})=z(x) u(x, \mathcal{I}) & \text { dans } \mathbf{R}^{N}\end{cases}
$$

Alors $u \geq w$ dans $\mathbf{R}^{N} \times[\mathcal{I}, T)$.

Démonstration. - Soit $z \in C_{o}^{\infty}\left(\mathbf{R}^{N}\right), w(\cdot, \mathcal{I})$ est à support compact dans $\mathbf{R}^{N} ;$ d'après la proposition 1.3 , il existe $R \in \mathbf{R}_{*}^{+}$tel que pour tout $t \in[\mathcal{I}, T)$, Supp $w(\cdot, t) \subset B_{R}(0)$. Et par conséquent le problème (1.31) est équivalent à :

$$
\begin{cases}w_{t}=\operatorname{div}\left[|\nabla w|^{p-2} \nabla w\right] & \text { dans } \left.\left.S_{R}=B_{R}(0) \times\right] \mathcal{I}, T\right), \\ w(\cdot, \mathcal{I})=z u(\cdot, \mathcal{I}) & \text { dans } B_{R}(0) .\end{cases}
$$

Comme $w(x, \mathcal{I})=0$ pour tout $|x|=R$, et $0 \leq z \leq 1$, alors $w \leq u$ sur $\partial S_{R}$. Ainsi d'après la proposition $1.1, w \leq u$ dans $\left.\left.\mathbf{R}^{N} \times\right] \mathcal{I}, T\right) \backslash S_{R}$; par conséquent $w \leq u$ dans $\mathbf{R}^{N} \times[\mathcal{I}, T)$.

\section{Le résultat principal}

Dans ce paragraphe, on va montrer l'existence et l'unicité de la trace initiale. Pour cela, on prouve l'inégalité faible d'Harnack suivante :

Proposition 2.1. - Soit u une solution faible dans $\bar{\Omega}_{T}$ de l'équation (1.1). Alors il existe une constante $C \in \mathbf{R}^{+}$dépendant de $N$ et $p$, telle que pour tout $x_{o} \in \mathbf{R}^{N}$ et pour tout $R \in \mathbf{R}_{*}^{+}$on a :

$$
\int_{B_{R}\left(x_{o}\right)} u(x, 0) d x \leq C\left\{R^{\frac{k}{p-2}} T^{-\frac{1}{p-2}}+T^{\frac{N}{p}} u^{\frac{k}{p}}\left(x_{o}, T\right)\right\}
$$

ò̀ $k=p+N(p-2)$.

Démonstration. - Soit $R \in \mathbf{R}_{*}^{+}$fixé, on pose

$$
V(x, t)=R^{\frac{-p}{p-2}} T^{\frac{1}{p-2}} u(R x, T t) .
$$

Pour montrer la relation (2.1), il suffit de prouver que

$$
\int_{B_{1}\left(x_{o}\right)} V(x, 0) d x \leq C\left\{1+V^{\frac{k}{p}}\left(x_{o}, 1\right)\right\} .
$$


Il est évident que $V$ est une solution faible dans $\bar{\Omega}_{1}$ de l'équation (1.1). Ainsi l'inégalité (2.3) est une conséquence immédiate du Corollaire 1 de [2].

On donne le résultat principal suivant :

THÉORÈme 2.2. - Soit u une solution faible non négative de l'équation (1.1) dans $\Omega_{T}$. Alors il existe une mesure $\mu$ non négative de Borel dans $\mathbf{R}^{N}$, telle que, pour toute fonction $\varphi \in C_{o}^{\infty}\left(\mathbf{R}^{N}\right)$, on a :

$$
\lim _{t \rightarrow 0} \int_{\mathbf{R}^{\mathbf{N}}} \varphi(x) u(x t) d x=\int_{\mathbf{R}^{N}} \varphi(x) \mu(d x) .
$$

De plus, il existe une constante $C$ dépendant de $N$ et $p$ telle que, pour tout $R \in \mathbf{R}_{*}^{+}$et pour tout $x_{o} \in \mathbf{R}^{N}$, on $a$ :

$$
\int_{B_{R}\left(x_{o}\right)} \mu(d x) \leq C\left\{R^{\frac{k}{p-2}} T^{-\frac{1}{p-2}}+T^{\frac{N}{p}} u^{\frac{k}{p}}\left(x_{o}, T\right)\right\} .
$$

Avant de donner la démonstration, on a besoin d'un certain nombre de résultats préliminaires. Pour cela, on prouve la

Proposition 2.3. - Soit u une solution faible non négative dans $\bar{\Omega}_{T}$ de l'équation (1.1). Soient $a$ et $b$ deux constantes telles que:

$$
0<a<\int_{B_{1}\left(x_{0}\right)} u(x, 0) d x \leq b
$$

Alors pour chaque $\epsilon \in \mathbf{R}_{*}^{+}$, il existe une constante dépendant de $N, p, b, \epsilon$ et $T$ telle que

$$
\int_{B_{1+\epsilon}\left(x_{o}\right)} u(x, t) d x \geq a, \text { pour tout } t \in[0, \mathcal{I}]
$$

Avant de prouver la proposition, on donne le

LEMME 2.4. - Soit u une solution faible non négative dans $\mathbf{R}^{N} \times[0, T)$ de l'équation (1.1); de plus on suppose que Supp $u(\cdot, 0)=H$ est compact. Soit $\pi$ un hyperplan de $\mathbf{R}^{N}$ ne rencontrant pas $H$. Alors pour tout $x \in \mathbf{R}^{N}$ tel que son symétrique $y$ par rapport à $\pi$, appartient au même demi-espace que $H$, on a

$$
u(x, t) \geq u(y, t), \text { pour tout } t \in[0, T)
$$




\section{A. Gmira}

Démonstration. - Puisque l'équation (1.1) est invariante par rotation et translation, on peut prendre comme repère celui qui est constitué par l'hyperplan $\pi$ et son orthogonal. Ainsi l'inéquation (2.8) devient:

$$
u\left(x_{1}, \cdots, x_{N}, t\right) \geq u\left(x_{1}, \cdots, x_{N-1},-x_{N}, t\right),
$$

pour tout $t \in[0, T)$ avec $x_{N}>0$.

Sans perdre la généralité, on peut supposer que $H \subset\left\{\left(y, x_{N}\right) ; y \in \mathbf{R}^{N-1}\right.$ et $\left.x_{N}>0\right\}$. Soient $x=\left(x_{1}, \cdots, x_{N}\right)$ et $y=\left(x_{1}, \cdots, x_{N-1}\right)$.

On pose $V(x, t)=u\left(y,-x_{N}, t\right)$. Alors

$$
V(y, 0, t)=u(y, 0, t), \text { pour tout } y \in \mathbf{R}^{N-1} \text { et } t \in[0, T) .
$$

D'autre part

$$
V\left(y, x_{N}, 0\right)=u\left(y,-x_{N}, 0\right)=0 \leq u\left(y, x_{N}, 0\right) .
$$

Ainsi en utilisant la proposition 1.2 , puisque $u(\cdot, t)$ et $V(\cdot, t)$ sont à support compact, on déduit

$$
u\left(y,-x_{N}, t\right)=V\left(y, x_{N}, t\right) \leq u\left(y, x_{N}, t\right), \text { pour tout } t \in[0, T)
$$

c'est-à-dire que l'on a bien la relation (2.9).

Lemme 2.5.- Soit u une solution faible dans $\bar{\Omega}_{T}$, non négative de l'équation (1.1) qui est à support compact. Soit $K \in \mathbf{R}^{+}$tel que

$$
\int_{\mathbf{R}^{N}} u(x, 0) d x \leq K .
$$

Si Supp $\mathrm{u}(\cdot, 0) \subset \mathrm{B}_{1}\left(\mathrm{x}_{0}\right)$, alors il existe une constante $C$ ne dépendant que de $N$ telle que

$$
u(x, t) \leq \frac{C K}{\epsilon^{2 N-1}}, \text { dans }\left\{\mathbf{R}^{N} \backslash B_{1+\epsilon}\left(x_{o}\right)\right\} \times[0, T],
$$

pour tout $\epsilon \in \mathbf{R}_{+}^{*}$.

Démonstration. - On suppose que $x_{o}=0$.

Soit $y_{o} \in \mathbf{R}^{N} \backslash B_{1+\epsilon}(0)$ fixé, on pose

$$
\begin{gathered}
S\left(y_{o}\right)=\left\{x \in \mathbf{R}^{N} /\left|y_{o}-x\right|<\frac{\epsilon}{2} \text { et } \cos \theta \geq \frac{1+\epsilon / 4}{1+\epsilon}\right\}, \\
-324-
\end{gathered}
$$


où $\theta$ est l'angle compris entre $y_{o}-x$ et $y_{o}$.

On note par $\pi\left(y_{o}, x\right)$ l'hyperplan formé par l'ensemble des points qui sont équidistants de $y_{o}$ et de $x$. Ainsi la distance $d$ de $\pi\left(y_{o}, x\right)$ à l'origine est donnée par :

$$
\left\{\begin{array}{l}
d=\min \|x\|, \text { sous la contrainte } \\
\left|y_{o}-x\right|-c_{1}=\epsilon / 2 . \\
\frac{\left\langle y_{o}-x, y_{o}\right\rangle}{\left\|y_{o}-x\right\|\left\|y_{o}\right\|}+C_{2}=\frac{1+\epsilon / 4}{1+\epsilon} \\
c_{2}>0, c_{1}>0
\end{array}\right.
$$

D'où $d=\left|y_{o}\right| \cos \theta-\frac{1}{2}\left|y_{o}-x\right|$.

Ainsi

$$
d \geq \frac{1+\epsilon / 4}{1+\epsilon}(1+\epsilon)-\epsilon / 4=1 .
$$

Par conséquent, si $x \in S\left(y_{o}\right)$, alors $x$ et $B_{1}(0)$ sont dans le même demiespace. D'après le lemme $2.4, u(x, t) \geq u\left(y_{o}, t\right)$, pour tout $t \in[0, T]$. D'autre part

$$
\begin{aligned}
K \geq \int_{\mathbf{R}^{N}} u(x, 0) d x=\int_{\mathbf{R}^{N}} u(x, t) d x & \\
& \geq \int_{S\left(y_{o}\right)} u(x, t) d x \geq\left|S\left(y_{o}\right)\right| u\left(y_{o}, t\right) .
\end{aligned}
$$

Et du fait que $S\left(y_{o}\right)=C(N) \epsilon^{2 N-1}$, alors on obtient bien la relation (2.14).

Lemme 2.6. - Soit u vérifiant les hypothèses du lemme 2.5. Pour tout $\epsilon>0$, il existe une constante $\mathcal{I}$ dépendant de $\epsilon, N, p, K$ et $T$ telle que l'on a la relation suivante :

$$
\text { Supp } \mathrm{u}(\cdot, \mathrm{t}) \subset \mathrm{B}_{1+\epsilon}\left(\mathrm{x}_{\mathrm{o}}\right), \text { pour tout } \mathrm{t} \in[0, \mathcal{I}] .
$$

Démonstration. - On considère la solution $u_{\lambda}$ dont l'expression est donnée par (1.25). Soit $\epsilon \in \mathbf{R}_{*}^{+}$donné, alors il existe deux constantes $\left.t_{o} \in\right] 0,1\left[\right.$ et $\lambda \in \mathbf{R}^{+}$dépendantes de $N, p, \epsilon, K$ et $T$ telles que l'on a :

$$
\operatorname{Supp} u_{\lambda}\left(\cdot, t_{o}\right)=\bar{B}_{1+\epsilon / 2}\left(x_{o}\right)
$$

et

$$
\min _{\substack{t \in[0, T] \\|x|=1+\epsilon / 4}} u_{\lambda}\left(x, t+t_{o}\right) \geq \frac{C K}{\epsilon^{2 N-1}}
$$




\section{A. Gmira}

où $C$ est la constante qui intervient dans la formule (2.14). En utilisant le lemme 2.5 , on obtient

$$
u(x, t) \leq u_{\lambda}\left(x, t+t_{o}\right) \text { sur } \partial B_{1+\epsilon}\left(x_{o}\right) \times\left[0, T^{\prime}\right] .
$$

D'autre part, puisque Supp $u(\cdot, 0) \subset B_{1}\left(x_{o}\right)$, alors

$$
u(x, 0) \leq u_{\lambda}\left(x, t_{o}\right) \text { dans } \mathbf{R}^{N} \backslash B_{1+\epsilon / 4}\left(x_{o}\right) .
$$

Par conséquent

$$
u(x, t) \leq u_{\lambda}\left(x, t+t_{o}\right) \text { dans }\left[\mathbf{R}^{N} \backslash B_{1+\epsilon / 4}\left(x_{o}\right)\right] \times[0, T] .
$$

Et pour déduire la formule (2.19), il suffit de choisir $\mathcal{I}$ (qui existe) telle que

$$
\text { Supp } \mathrm{u}_{\lambda}\left(\cdot, \mathcal{I}+\mathrm{t}_{\mathrm{o}}\right)=\overline{\mathrm{B}}_{1+\epsilon}\left(\mathrm{x}_{\mathrm{o}}\right) \text {. }
$$

Après les 3 lemmes, on est en mesure de donner la démonstration de la proposition 2.3.

Preuve de la Proposition 2.3. - On suppose que $x_{o}=0$. Soit $\alpha \in \mathbf{R}^{+}$fixé tel que

$$
0<a<\alpha<\int_{B_{1}\left(x_{o}\right)} u(x, 0) d x \leq b .
$$

Il existe deux constantes $d_{1}$ et $d_{2}$ telles que :

$$
\left\{\begin{array}{l}
0<d_{1}<d_{2}<1 \\
\int_{B_{d_{1}}(0)} u(x, 0) d x=a \\
\int_{B_{d_{2}}(0)} u(x, 0) d x=\alpha
\end{array}\right.
$$

Soit $v \in C^{\infty}\left(\mathbf{R}^{+}\right)$décroissante définie par :

$$
v(r)= \begin{cases}1 & \text { pour } 0 \leq r \leq d_{1} \\ 0 & \text { pour } r \geq d_{2}\end{cases}
$$

On considère la solution faible dans $\mathbf{R}^{N} \times[0, T]$ de l'équation (1.1), dont la donnée initiale est :

$$
\begin{gathered}
w(x, 0)=v(|x|) u(x, 0) \leq u(x, 0) . \\
-326-
\end{gathered}
$$




\section{Existence de la trace initiale}

D'après la proposition $1.1, w(x, t) \leq u(x, t)$ dans $\bar{\Omega}_{T}$. D'autre part d'après le lemme 2.6 et du fait que

$$
\int_{\mathbf{R}^{N}} w(x, 0) d x=\int_{B_{d_{2}}(0)} w(x, 0) d x \leq \int_{B_{d_{2}}(0)} u(x, 0) d x \leq \alpha ;
$$

pour tout $\epsilon \in \mathbf{R}^{+}$il existe une constante $\mathcal{I}$ telle que

$$
\text { Supp } \mathrm{w}(\cdot, \mathrm{t}) \subset \mathrm{B}_{1+\epsilon}(0), \text { pour tout } \mathrm{t} \in[0, \mathcal{I}] .
$$

Ainsi d'après la relation (2.28), on obtient :

$$
\int_{B_{d_{2}}(0)} w(x, 0) d x=\int_{\mathbf{R}^{N}} w(x, 0) d x=\int_{\mathbf{R}^{N}} w(x, t) d x=\int_{B_{1+\epsilon}(0)} w(x, t) d x .
$$

Par conséquent, on a

$$
\begin{aligned}
\int_{B_{1+\epsilon}(0)} u(x, t) d x & \geq \int_{B_{1_{+\epsilon}}(0)} w(x, t) d x=\int_{B_{d_{2}}(0)} w(x, 0) d x \\
& \geq \int_{B_{d_{1}}(0)} w(x, 0) d x=\int_{B_{d_{1}}(0)} u(x, 0) d x=a
\end{aligned}
$$

ceci pour tout $t \in[0, \mathcal{I}]$, ce qui prouve la relation (2.7).

Démonstration du théorème 2.2. - La démonstration du théorème va se faire en 2 étapes. Dans la lère étape, on prouve l'existence; quant à l'unicité, elle va se démontrer dans la 2ème partie.

1ère étape : l'existence.

D'après la proposition 2.1 , pour tout $s \in] 0, T$ [ et pour tout $R \in \mathbf{R}^{+}$, on a :

$$
\int_{B_{R}\left(x_{o}\right)} u(x, s) d x \leq C\left\{R^{k / p-2}(T-s)^{-1 / p-2}+(T-s)^{N / p} u^{k / p}\left(x_{o}, T\right)\right\}
$$

où $C$ est une constante indépendante de $s$.

Donc il existe une suite $\left(s_{n}\right)_{n \in N}$ telle que $s_{n} \searrow 0$ et $u\left(\cdot, s_{n}\right)$ converge faiblement vers une mesure $\mu$. Ainsi, pour toute fonction $\varphi \in C_{o}\left(\mathbf{R}^{N}\right)$ on a :

$$
\lim _{n \rightarrow+\infty} \int_{\mathbf{R}^{N}} \varphi(x) u\left(x, s_{n}\right) d x=\int_{\mathbf{R}^{N}} \varphi(x) \mu(d x) .
$$

Par conséquent l'inégalité (2.4) est bien vérifiée. Et du fait que $u \geq 0$, alors $\mu$ est positive. A priori la mesure $\mu$ dépend de la suite $\left(s_{n}\right)_{n \in N}$; mais en fait on va démontrer qu'il n'en est rien, dans l'étape suivante : 


\section{A. Gmira}

2ème étape : l'unicité.

Soit $t \in(0, T / 2)$. D'après la proposition 2.1 , on a :

$$
\int_{B_{1}\left(x_{o}\right)} u(x, t) d x \leq C\left\{\left(\frac{2}{T}\right)^{k / p-2}+T^{N / p} u^{k / p}\left(x_{o}, T\right)\right\} .
$$

Puisque $u$ est non identiquement nulle, on peut supposer que $\int_{B_{1}\left(x_{o}\right)} u(x, t) d x \neq 0$. Soient $\epsilon, d \in \mathbf{R}^{+}$et $\alpha \in(0, T / 4)$, en utilisant la proposition 2.3 , il existe $\mathcal{I} \in \mathbf{R}_{*}^{+}$telle que

$$
\int_{B_{1+\epsilon}\left(x_{o}\right)} u(x, t+\alpha) d x+d \geq \int_{B_{1}\left(x_{o}\right)} u(x, \alpha) d x .
$$

Ceci pour tout $t \in[0, \min (T / 4, \mathcal{I})]$.

Supposons que l'on a deux suites $\left\{s_{n}\right\}_{n \in \mathbb{N}}$ et $\left\{s_{n}^{\prime}\right\}_{n \in N}$ telles que $u$ converge faiblement vers une mesure $\mu_{1}$ quand $s_{n}$ tend vers 0 (respectivement vers $\mu_{2}$ quand $s_{n}^{\prime}$ tend vers 0 ). Du fait que les deux mesures vérifient la relation (2.4), elles sont régulières. Pour $t$ fixé, soit $s_{n}=\alpha$; en passant à la limite sur $n$ dans la relation (2.37), on obtient :

$$
\int_{B_{1+\epsilon}\left(x_{o}\right)} u(x, t) d x+d \geq \int_{B_{1}\left(x_{o}\right)} \mu_{1}(d x) .
$$

En prenant $t=s_{n}^{\prime}$ et en passant à la limite sur $n$, on a

$$
\int_{B_{1+\epsilon}\left(x_{o}\right)} \mu_{2}(d x+d) \geq \int_{B_{1}\left(x_{o}\right)} \mu_{1}(d x),
$$

ceci pour tout $\epsilon, d \in \mathbf{R}^{+}$, par conséquent

$$
\int_{B_{1}\left(x_{o}\right)} \mu_{2}(d x) \geq \int_{B_{1}\left(x_{o}\right)} \mu_{1}(d x) .
$$

En inversant les rôles de $t$ et $\operatorname{de} \alpha$, on déduit

$$
\int_{B_{1}\left(x_{o}\right)} \mu_{2}(d x)=\int_{B_{1}\left(x_{o}\right)} \mu_{1}(d x)
$$

c'est-à-dire que $\mu_{2}\left(B_{1}\left(x_{o}\right)\right)=\mu_{1}\left(B_{1}\left(x_{o}\right)\right)$.

Or pour tout $\lambda \in \mathbf{R}$ et pour tout $x \in \mathbf{R}^{N}$, on a :

$$
\mu_{i}\left(\lambda\left(x-x_{o}\right)\right)=|\lambda| \mu_{i}\left(x-x_{o}\right) ; i=1,2 .
$$


Existence de la trace initiale

Par conséquent $\mu_{1}=\mu_{2}$ dans $\mathbf{R}^{N}$.

Ce qui achève la démonstration du théorème.

\section{Références}

[1] Aronson (D.G.). - Widder's inversion theorem and the initial distribution problem, S.I.A.M. J. Math. Anal., t. 12, 1981, p. 639-651.

[2] Dibenedetto (E.). - Intrinsic Harnack type inequalities for solutions of certain degenerate parabolic equations, to appear.

[3] Dibenedetto (E.) and Friedman (A.). - Regularity of solutions of nonlinear degenerate parabolic systems, J. Reine Angew. Math., t. 349, 1984, p. 83-128.

[4] LEE (S.V.) and AMES (W.F.). - Similarity solutions for non-Newtonian fluids, A.T. Ch. E. Journal, t. 12, 4, 1966, p. 700-708.

(Manuscrit reçu le 2 mars 1987) 